\title{
Exploring the motivations involved in context aware services
}

\author{
Chris Roast \\ Culture, Communication and Computing Research Institute \\ Sheffield Hallam University \\ 153 Arundel Street \\ Sheffield, S1 2NU \\ United Kingdom \\ c.r.roast@shu.ac.uk
}

\author{
Xiaohui Zhang \\ Culture, Communication and Computing Research Institute \\ Sheffield Hallam University \\ 153 Arundel Street \\ Sheffield, S1 2NU \\ United Kingdom \\ xiaohui.zhang2@student.shu.ac.uk
}

\begin{abstract}
This paper reports on research focused upon understanding the factors influencing the effective use of context aware adaptive systems. Unlike many desktop applications, ubiquitous computing supports users in dynamic situations by utilizing surrounding context to help them manage and utilise technology. It is by its nature highly dynamic since it responds to changes in context of use, and this brings new challenges to interaction design. In particular, there is still little research into human factors relating to the effectiveness and appropriateness of ubiquitous computing concepts. We review theoretical factors regarding human user's motivation, emotion, perception and preference that are relevant to evaluating ubiquitous computing. Here we then report on empirical research relating these theoretical factors to the use of contextually aware adaptive systems. The results show that there is a significant difference in users' preferences between intrinsic and extrinsic motivations. The other findings identify the importance and role of user involvement in decision-making processes. Overall the work raises interesting questions about the nature of empirical research as a methodology of relevance to adaptive system design.
\end{abstract}

Keywords: Adaptive systems; Context aware applications; User centred design; Empirical Studies.

\section{ADAPTIVE SYSTEM RESEARCH}

Along with the development of microchip and sensing technologies, more and more ContextAware applications have been introduced to our daily lives. The use of GPS, sensors and wireless connectivity provides more and more opportunities for data rich responsive applications, integrated with a variety of products. Many of the applications have been proposed to improve the quality of life, and furthermore, to engage the end users with a richer or augmented environment. Therefore, as Strand and Linnhoff-Popien (2004) stated that context dependency is a major issue in recent research work in the area of ubiquitous computing systems. Accordingly, although many researchers bring innovative ideas to the field of ubiquitous computing, there is still a lack of human factors research in many projects, which may finally decrease the envisaged user acceptance and adoption.

Unlike desktop applications, context aware computing supports users in dynamic situations by utilizing surrounding context. Contextual information is monitored and when it changes the application or device configuration is changed to suit the new situation. For example, some GPS navigators will automatically generate a new route of the shortest distance when the user has walked away from the preset one. In this example, the shortest distance has been used as the only indicator of adaptation. Similarly, adaptation strategies for Quality of Service have been used by many Context-Aware applications to generate alternative reconfigurations when a change in the context occurs. Besides system and environmental components, users' internal states may be also changeable in different situations, mainly including their motivation, emotion, perception and preference. The question of interest to us is do such automated methods help users when designers wish to take account of human factors like an unplanned decision. For instance, in the above driving example, it would be less thoughtful to recreate the new shortest route automatically in a situation where that the driver simply stops for a break at a local diner.

This research takes a user's perspective, which focuses on user's motivation, emotion, perception and preference in dynamic situations. The results of proposed experiment were expected to show the effects of different motivations on user's preference 
in different context-aware adaptation plans, in order to demonstrate the importance of user involvement in the decision-making process for context-aware adaptation. This is of particular importance for the majority of systems that at best can only roughly estimate the users' motivational state. This research was also expected to demonstrate a critical understanding of human factor issues for context-aware services, in order to help understand the role that user involvement plays, and the shifting of research focus from interaction between user and system to a broader concept of contextaware activities. Correspondingly, releasing users from heavy interaction may help to enhance their subjective experiences, as Norman (1988, p.186) proposed that "The computer is invisible, hidden beneath the surface; only the task is visible".

\section{MOTIVATION, PERCEPTION, SUBJECTIVE EMOTION AND PREFERENCE}

This section introduces some basic theories of motivation, perception, subjective emotion and preference, and how they relate to $\mathrm{HCl}$. It provides a theoretical basis for the proposed study that aims to understand the complex human factor issues in context-aware services.

Psychologically, motivation can be classified into two types: Intrinsic and Extrinsic. Intrinsic motivation refers to motivation that is driven by pleasure or satisfaction that comes from the task itself, and Extrinsic motivation generally refers to activities that could only be prompted from outside of the individual, such as reward or punishment. An important development closely related to this is the Reversal theory, created and developed by Smith and Apter in the mid-1970s. This early work led to the description of a dynamic system, based on the concept of the "reversal:" the switching between opposed motivational states. For example, you can eat being serious and pursuing a goal in eating, or being in a spirit of seeking pleasure in the moment. Thus, "serious, future-oriented" and "playful, focused on the present" are two possible (and opposite) motivational states that can be associated with the activity of eating. One key aspect of Reversal theory is that only one end of the opposite states can be active at a time.

In the field of $\mathrm{HCl}$ research, Hassenzahl (2003, 2008) suggested that people perceive interactive products along two different dimensions, which are Pragmatic quality - refers to the fulfilment of taskrelated "do-goals" (e.g. make a phone call, usability) and non-instrumental Hedonic quality - refers to the fulfilment of "be-goals" (e.g. novelty, beauty or being competent). He also suggested that the perceived quality of interactive products heavily depends on features of the context of use, such as whether an individual has to perform a specific task or not (2003). Hassenzahl and his colleagues (2008, 2010) have conducted a series of studies showing that the user's preference for different music players can be influenced by preset scenarios. They further suggested that hedonic quality is a "motivator", capturing: (i) the product's perceived ability to create positive experiences through need fulfilment and (ii) a "hygiene factor", enabling the fulfilment of needs through removing barriers but not being a source of positive experience in itself. This is related to an earlier theory proposed by Maslow (1954), suggesting that deficiency need is a need which creates a negative feeling if not fulfilled, but does not contribute much to a positive feeling.

In addition to motivational state, user's perception can also be influenced by long term issues such as personal historical experience. Humans construct perceptions drawing both on sensations coming bottom-up to the brain and on our experience and expectations (top-down effect). One example is conditioned response. At a very basic level, Human preferences and behaviours can be manipulated by Primary Reinforcers (e.g. getting food when hungry) and Conditioned Reinforcers (e.g. money, good grades). Conditioned Reinforcers get their power through learned association with primary Reinforcers - each of which has been linked with more basic rewards and we only respond to a conditioned stimulus if we have already learnt it (Myers 2010, p. 307).

Our personal historical experience also plays an important role in the generation of subjective emotional experience. According to the TwoFactors Theory that proposed by Stanley SchAchter and Jerome Singer (1962), our physiology and our cognitions - perceptions, memories, and interpretations - together create emotion. In this theory, emotions therefore have two ingredients: physical arousal and a cognitive label. As a further development of some early theories, Schachter and Singer presumed that our experience of emotion grows from our awareness of our body's arousal. They also believed that emotions are physiologically similar. Thus, in their view, an emotional experience requires a conscious interpretation of the arousal (Myers 2010, p. 498). Our personal historical experience, such as culture, education and society, largely determines which cognitive label we will take for a situation like or dislike, impressed or disappointed, and so on.

Overall, our awareness of surroundings, understanding of situations, preference between selections and subjective emotion, are greatly affected by our cognition. But the influencing factors, such as situational contexts and personal historical experience are difficult to detect and assess. Therefore it is worth considering user 
engagement in context-aware adaptation especially where context-aware services which aim to enhance subjective user experience in dynamic situations. Furthermore, a deeper understanding of the human factor issues involved in a contextaware activity may help develop more effective interaction techniques for facilitating user involvement. As a simple example, the parameters that have been identified as less influential in the users' decision making could be moved to the advanced options, in order to simplify the user interface design.

\section{METHODOLOGY}

These theories provide a basis for a study to examine the motivation and interaction with adaptive systems. The two-phase study was developed to in the domain of location-based services. In recent years, many research efforts have focused on Location-based applications, as Häkkilä et al. (2009) stated that "Location-based applications form a significant area within mobile context-awareness, and have great potential for future commercial applications; this is particularly relevant with today's GPS-enabled mobile devices with mobile maps. Common applications demonstrating location-awareness are tour guides in the city, campus or museum environments, shopping assistants, messaging systems, and location-sensitive reminders".

The study consisted of two phases. The phase one study used a focus-group discussion to preliminarily investigate the effects of different motivations on people's preferences in wayfinding activities. The findings have been studied and used to conduct the following experiment.

For the phase two study, a controlled betweengroup experiment was designed to show the difference of users' preference in wayfinding activities between intrinsic and extrinsic motivations, in order to further study the human factors in the domain of location-based services. A specific context aware scenario and prototype were developed according to the objective, based on HP Mediascape (DfES and HP labs), to provide a realistic setting for research subjects.

\subsection{Factor identification - Phase 1}

Four participants from the investigator's social and research networks attended the focus-group discussion. At first, the investigator explained the definition of wayfinding activities, and then asked the participants to write down a recent experience of walk or travel. Also, each participant was required to identify and name at least three factors that influenced their choice of different routes. A short review of all reported experiences had been undertaken by the investigator to classify the activities according to different motivational states. Two of the four respondents reported a recent experience of walking to office and the other half were about travelling to strange places. Both types of experience were discussed, with guiding questions to lead the discussion based on the following three dimensions:

(i) Motivational states - e.g. "Do you think it's important?", "Do you feel like you have no choice but to do so?"

(ii) Subjective Experience - e.g. "Did you enjoy it?", "How do you feel when (activities)?"

(iii) Influencing Factors - e.g. "What influenced your choice?"

In summary, the discussion showed a possible relationship between people's motivational states and preferences in wayfinding activities - the more purposeful an activity is, the less likely the people will enjoy and engage into it, and the less likely the hedonic factors will influence their preferences and behaviours. As a result, ten major factors influencing wayfinding activities were identified:

(i) Safety was agreed to be the primary influencing factor.

(ii) Time limitation.

(iii) Distance - usually related to time limitation and physical capabilities.

(iv) Weather Condition.

(v) Surroundings - as the participants stated, the desire to see "new", "interesting" or "beautiful" things can be a very strong motivating force.

(vi) Cost - refers to transport fares.

(vii) Physical capabilities - refer to two aspects. Firstly, as one participant explained "when my kids were small they can't walk too far". Secondly, it's also related to people's subjective experience, as one participant stated "if two hours is too long then you are not going to enjoy the walk".

(viii) Convenient - mainly refers to road quality.

(ix) Social factors - refer to two aspects: the people that directly involved in same activity and the social influence of ongoing activities.

(x) Knowledge or Familiarization - related to various issues, such as direction, safety and landscape. According to the participants' reports, the effects of knowledge can be quite different from one another - on one hand, knowledge may help reduce people's negative feelings like nervous or fear, as one participant reported "if you plan well, you don't get nervous", "safety may coming to it then you do this journey twenty times and something else coming". But on the other hand, knowledge 
will also reduce the attraction of "new things".

\subsection{Measures}

According to the study objectives, the Situational Motivation Scale (SIMS, Guay et al., 2000) and the Pleasure-Arousal-Dominance emotion scales (Mehrabian and Russell, 1974) had been chosen to assess the participants' motivational and emotional states before and after the given task. Also, a questionnaire based on 7-point Likert scale had been used to evaluate how much participants' selections been influenced by the previous identified influencing factors.

\subsection{Study Design - Phase 2}

During the experimental process, all participants were asked to carry out an outdoor navigation task individually with a provided GPS navigator (developed based on Windows-Mobile PDA and HP Mediascape). In order to guide the participants into intrinsic and extrinsic motivational states, two different experimental settings had been designed according to several aspects. Firstly, the investigator explained some selected background information to the extrinsic group to convince the participants that the experiment is important. Then they were given a clear goal which asked them to complete the Navigation task in 25 minutes. Correspondingly, the intrinsic group was only been asked to follow the route that was presented by the GPS navigator. Secondly, as stated before that intrinsic motivation refers to motivation that is driven by pleasure or satisfaction that comes from the task itself, and extrinsic motivation generally refers to activities that could only be prompted from outside of the individual such as reward or punishment. Accordingly, for the intrinsic group, a payment of 20 pounds was given to the participant before the task was started. For the extrinsic group, the payments were given after the task was completed. Thirdly, the extrinsic group were given interruptions to reduce the likelihood of the close engagement with the task as one would expect with intrinsic motivation. Interruptions served the purpose of undermining confidence in task performance. Operationally the GPS navigator would ask the extrinsic group to stop for a few seconds when they reach the preset locations (two interruptions before they made the choices of routes and one time after).

During the walk, participants were asked to choose between two different routes provided by the Navigator. According to the results of previous focus-group study, the major influencing factors that affect people's route selection are safety, distance, landscape and convenience. So, for each of the routes, a few texts and three landscape photos had been used to represent those factors to the participants. The Route One was expected to be perceived as a short and convenient way by the participants; it was taken a distance of 580 meters mainly through narrow streets (without many pedestrians). The Route Two was expected to be perceived in direction opposite to the route one long, inconvenient but safe and beautiful; it was taken a distance of 750 meters that through a public garden and the main pedestrianised street of the city.

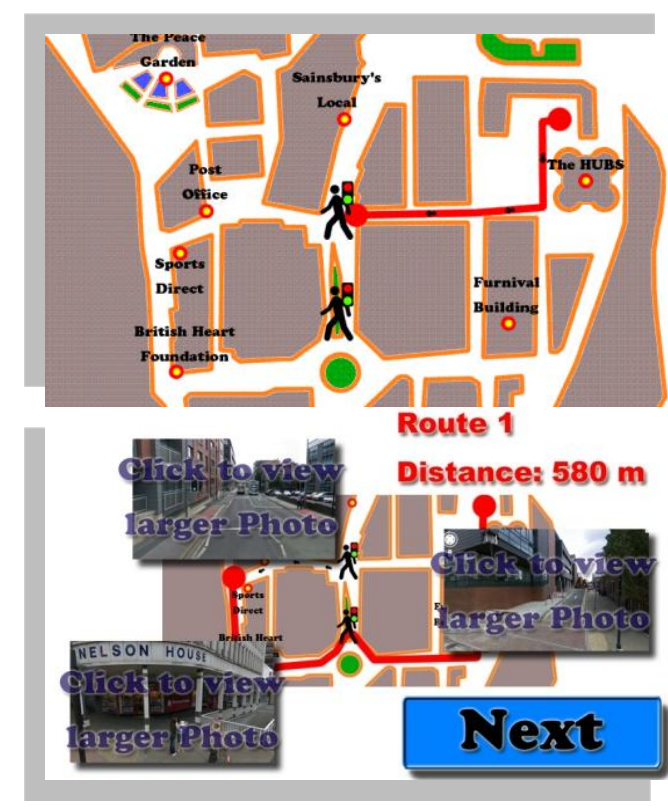

Two new Routes Have been found!

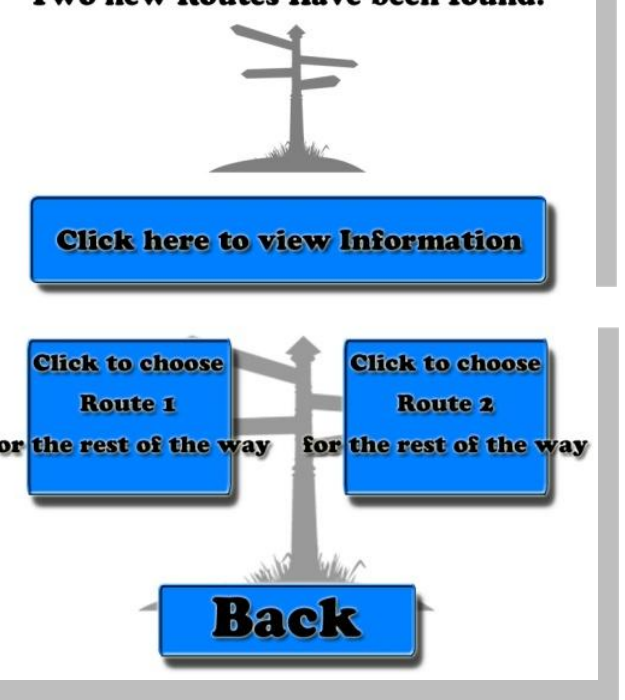

Figure 1: Screenshots of the GPS Navigator 


\section{THE STUDY}

Twenty participants were recruited and randomly assigned to two groups. All participants were students (both undergraduate and postgraduate) from the Sheffield Hallam University and the University of Sheffield between the ages of 20 to 40 , including 13 male and seven female. All international students had 6.5 IELTS, or equivalent, and had studied in UK for at least one and half years. The total study duration was two weeks and all experiments were done between $11 \mathrm{am}$ and $1 \mathrm{pm}$.

\subsection{Results}

\subsubsection{Motivations}

The Wilcoxon signed-rank test showed that for the Intrinsic Group, Intrinsic Motivation levels were significantly higher on Post test than on Pre test, $z$ $=-2.818, p<.05, r=-.63$; For Extrinsic Group, there was no significant difference in Intrinsic Motivation levels between Post test and Pre test have been found. However, there was also no significant change in extrinsic motivational state (Integrated Motivation and External Motivation) for both groups.

\subsubsection{Preferences}

The Mann-Whitney test showed that there were significant differences in two of the influencing factors between groups including Time Pressure $(z$ $=-2.330, p<.05)$ and subjective Body State $(z=-$ $2.337, p<.05)$. The results clearly indicated that the extrinsic group had been influenced by goalrelated factors (Time Pressure) more than the intrinsic group. An interesting finding is the different influences of subjective Body State between groups. The possible reason, as Csíkszentmihályi $(1975,1993)$ stated that a lack of awareness of bodily needs is one important factor for the experience of flow - the increase of intrinsic motivation leads to a decreased influence of awareness of general body state.

\subsubsection{Subjective Emotions}

The Wilcoxon signed-rank test showed that there were significant changes in Arousal level before and after the task for both Groups $(p<.05)$. However, there was no significant change in Pleasure and Dominant level found. The results of post-surveys also showed that half the participants in extrinsic group were happier after they completed the tasks. According to the Two-Factor theory, a possible reason for this is that their arousal levels had been improved by the task - it appears unlikely that the experimental setting was able to change the participants' cognitive labels.

\subsubsection{Other Findings}

Another interesting finding is that the MannWhitney test showed that there was a significant difference in Number of Clicks between groups $(z=$
-2.256, $p<.05)$ - the extrinsic group spend more time and efforts on the information that provided by the GPS navigator, which was totally against to the initial expectation. This finding implies that the experimental scenario made the extrinsic group pay more attention to the task by convincing them that the experiment is important.

\subsection{Discussion and Reflection}

The implemented empirical study indicated the different user preferences and behaviours in different situations. However, the experimental setting for extrinsic group was rather weak and it appears that it did not always lead to an extrinsic motivational state. For example, half the extrinsic group reported being influenced by Landscapes by contrast, whereas less than half reported being influenced by Time Pressure. Some of the participants believed that the longer route would be easier and faster to go through but at least two of the participants who picked the longer route were just wanted to see the city's Peace Garden - This was rather surprising since the investigator had carefully explained to them how important it is to complete the task as soon as possible. One possible basis for this lack of extrinsic motivation is the argument of Zimbardo and Gerrig (2002, p. 328 ). They propose that motivation is not derived from objective entities, but from our subjective explanations of such entities. According to this argument, the importance of the given goal in the implemented study was explained differently by the participants, and thus results in different users' preferences and behaviours.

As stated before, extrinsic motivation generally refers to activities that could only be prompted from outside of the individual such as reward or punishment. In the phase two study, both intrinsic and extrinsic groups were informed before the experiment that they will receive a payment of 20 pounds for their participation. The only difference is that the participants in extrinsic group had received their payments after the task was completed. By the results, we can see that it was not enough to convince the participants that the given objective (complete the task as soon as possible) is important to them. An enhanced method was therefore developed, which aimed to improve the experiment efficiency for the extrinsic group. The implementation details and results will be reported in our future papers.

\section{CONCLUSIONS}

This research has mainly focused on the development of an empirical method, which aims to improve understanding of the complex human factor issues in context-aware services. The proposed empirical method had been evaluated in 
the domain of location-based services. Accordingly, the implemented two-phase empirical study has examined how the users' preferences can be influenced by different motivational states. The findings show that among extrinsic motivated users, the pragmatic factors that directly related to task and goal exerted a greater influence on their preferences and behaviours. By contrast, the intrinsic motivated users were usually more influenced by the non-instrumental hedonic factors, sometimes even the application itself (the GPS navigator) was ignored by the participants to some degree.

Another important factor determining the users' preferences is the individual perceptions, which refers to two aspects. Firstly, as stated before, motivation is not derived from objective entities, but from people's subjective explanations of such entities. In other words, different people may perceive the same situation differently, therefore be motivated differently. Secondly, users' perception of the same thing (event or physical/digital entity) may be different and based on factors such as personal historical experience. For example, in the implemented phase two study, even though the expected perceptions for the two different routes had been generally accepted by the participants, there were still some participants who believed that the longer route could be faster to go through. There were also two participants from the Intrinsic Group who reported that their choices of routes had mainly been influenced by judging narrow streets to be more attractive than the public garden.

Our suggestion is that for the context-aware application with the aim to enhance subjective user experience, the designer should carefully assess human factor issues relating to desired motivational states and employ research techniques developed and illustrated in this study. If there is a need of dynamic adaptation and the proposed application is unable to comprehensively assess the user's historical experience or current situation, then the user should be given control during the decisionmaking processes. Future work in this will involve clarifying the empirical approach we've shown to ensure it can serve as a general approach for future research in this area.

\section{REFERENCES}

Strang. T, Linnhoff-Popien. C. (2004) A Context Modeling Survey. UbiComp First International Workshop on Advanced Context Modelling, Reasoning and Management. pp. 34-41. Nottingham, England.

Norman, Donald A. (1988) The Design of Everyday Things. Doubleday. New York.

K. C. P. Smith and M. J. Apter (1970) Reversal Theory Society. Reversal Theory Society. http://www.reversaltheory.org/ (25 September 2011).

Hassenzahl, M. (2003) The thing and I: Understanding the Relationship between User and Product. From Usability to Enjoyment. pp. 31-42. Kluwer Academic Publishers.

Hassenzahl, M. (2008) User experience (user experience): Towards an Experiential Perspective on Product Quality. Proceedings of the 20th International Conference of the Association Francophone d'Interaction HommeMachine. pp.11-15. ACM, New York.

Hassenzahl. M, Schöbel. M, Trautmann. T. (2008) How motivational orientation influences the evaluation and choice of hedonic and pragmatic interactive products. Interacting with Computers, 20, 473-479.

Hassenzahl. M, Diefenbach. S, Göritz. A. (2010) Needs, Affect, and Interactive Products - Facets of User experience. Interacting with Computers, 22, 353-362.

Maslow, A.H. (1954) Motivation and Personality. Harper and Row, New York.

Myers, David G. (2010) Psychology, 9th Edition. Worth, New York.

Schachter, S. \& Singer, J. (1962) Cognitive, Social and Physiological Determinants of Emotional State. Psychology Review, 69, 379-99.

Häkkilä. J, Schmidt. A, Mäntyjärvi. A, Sahami. A, Åkerman. P, K. Dey. A. (2009) Context-Aware Mobile Media and Social Networks. Mobile $\mathrm{HCl}$ '09 Proceedings of the 11th International Conference on Human-Computer Interaction with Mobile Devices and Services. ACM, New York.

DfES and HP Labs. HP Mscape. http://www.hpl.hp.com/mediascapes (2 April 2011).

Guay, F., Vallerand, R.J., Blanchard, C. M. (2000) On the assessment of state intrinsic and extrinsic motivation: The Situational Motivation Scale (SIMS). Motivation and Emotion, 24, 175213.

Csikszentmihalyi, M \& Rathunde, K (1993) The measurement of flow in everyday life: Towards a theory of emergent motivation. Developmental perspectives on motivation. Nebraska Symposium on Motivation, 40, 57-97.

Csíkszentmihályi, Mihály (1975) Beyond Boredom and Anxiety: Experiencing Flow in Work and Play. Jossey-Bass, San Francisco.

Zimbardo, Philip G. \& Gerrig, Richard J. (2002) Psychology and Life, 16th Edition. Longman, New York. 